\title{
ANISOTROPIC FLUORESCENCE OF POLAR MOLECULES IN EXTERNAL ELECTRIC FIELD, DIPOLE MOMENTS OF PARA-DISUBSTITUTED TRANS-STILBENES IN THE FIRST EXCITED SINGLET STATE*广
}

\author{
A. Kawski and A. Kubicki \\ Luminescence Research Group, Institute of Experimental Physics, University of \\ Gdańsk, Wita Stwosza 57, 80-952 Gdańsk, Poland \\ (Received June 19, 1990)
}

The electric dipole moments $\mu_{e}$ in the lowest excited singlet state of nine short-lived para-disubstituted trans-stilbenes were determined experimentally based on the effect of the external electric field on the fluorescence anisotropy. The values of $\mu_{\mathrm{e}}$ obtained from Liptay's theory with the employment of the Onsager effective field are in satisfactory agreement with those calculated based on the solvatochromic effect. In the case of para-substituted trans-stilbenes with the same donor substituent $\mathrm{R}=\mathrm{N}\left(\mathrm{CH}_{3}\right)_{2}$ in position 4 and different acceptors $R^{\prime}$ in position $4^{\prime}$, the enhancing electron properties of the acceptor are accompanied by the increase in $\mu_{\mathrm{g}}$ - the dipole moment in the ground state, and $\mu_{e}-$ the dipole moment in the excited state. There exists a linear relation between the values of $\mu_{\mathrm{e}}$ and $\mu_{\mathrm{g}}$.

PACS numbers: $33.50 .-\mathrm{j}$

\section{Introduction}

When polar molecules with isotropically distributed transition moments (clasically, the so-called virtual linear electronic oscillators) are excited in solution by linearly polarized or natural light, anisotropic fluorescence, alternatively partial polarization of fluorescence, can in general be observed. This phenomenon is called

*This work was supported by CPBP 01.06.

tDedicated to Professor Michael Kasha on the occasion of his 70th birthday. 
photoselection. Fluoroscence anisotropy in solution depends on the mean lifetime $\tau_{\mathfrak{f}}$ of luminescent molecules and on the relaxation time $\tau_{\mathrm{r}}$ of their thermal rotational motion. For $\tau_{\mathrm{f}} \leq \tau_{\mathrm{r}}$, the emission anisotropy ${ }^{\ddagger}$ assumes considerable values as, for example, was observed for a number of trans-stilbene derivatives [4,5]. For $\tau_{\mathrm{f}} \gg \tau_{\mathrm{r}}$, complete fluoroscence depolarization due to Brownian rotational motions occurs in most cases. If the solution of polar molecules is placed in an external electric field, then, as a result of additional orientation of the dipole moments in the direction of the field applied, the emission anisotropy increases compared to that observed in the absence of the field. This effect was first detected by Czekalla $[6,7]$ and employed for the determination of electric dipole moments in excited states. According to the simplified Czekalla's theory, the mean lifetime $\tau_{\mathrm{f}}$ of the excited molecules is assumed to exceed markedly the rotational relaxation time $\tau_{\mathbf{r}}$ which results in complete depolarization of fluoroscence $(r \approx 0)$ in the absence of an external electric field. It is moreover assumed that the mean lifetime $\tau_{\mathrm{f}}$ of the excited molecules is high enough, compared to the dielectric relaxation time $\tau_{\varepsilon}\left(\tau_{\mathfrak{f}} \gg \tau_{\varepsilon}\right)$ for the fluorescence to occur from the state of statistical equilibrium between the excited molecules and their surroundings (solvent). Thus, the electric dipole moment $\mu_{\mathrm{e}}$ in the excited state is responsible for the increase in the fluorescence emission anisotropy caused by the external electric field.

In the cases in which the initial emission anisotropy is of a magnitude comparable to the emission anisotropy $r_{0}$ in the absence of the Brownian rotational motions, it is indispensable to take into account the rotational diffusion of molecules under the influence of the electric field. A theory including such diffusion effects was worked out by Weber [8].

The most general theory was developed by Liptay [6] who took into account the absorption and fluorescence band shifts due to the change in the electric dipole moments $\left(\mu_{\mathrm{e}}>\mu_{\mathrm{g}}\right)$, and the fact that the lifetime $\tau_{\mathrm{f}}$ of a luminescent molecule might be comparable to the dielectric relaxtion time $\tau_{\varepsilon}$.

In view of experimental difficulties few reports have up till now appeared [6, $7,10-12]$. The observed effects are slight and, therefore, the experiments necessitate the employment of a highly sensitive mesuring apparatus more so that the method of measurement (excitation at right angle to the observation direction) does not enable high fluorescence light intensity to be attained. It is for these reasons that Czekalla $[6,7]$ carried out measurements only for substances with long lifetimes, exceeding $1 \mathrm{~ns}$, and high emission yield. In the sixties, analogue measurement techniques were only available which required higher intensities of recorded light. Nowadays, the rapid development of technology enables these difficulties to be surmounted. In the present paper, we employed an apparatus for the measurement of the emission anisotropy by a fully automatic computer-aided single-photon

\footnotetext{
tEmission anisotropy, in the case of excitation of an isotropic luminophor by plane polarized light, is defined as [1] $r=\left(I_{\|}-I_{\perp}\right) /\left(I_{\|}+2 I_{\perp}\right)$, where $I_{\| \mid}$denotes the intensity of the component of photoluminescence parallel to the electric vector of the primary light, $I_{\perp}$ - the intensity of the perpendicular component. Between the emission anisotropy $r$ and the degree of polarization $P=\left(I_{\|}-I_{\perp}\right) /\left(I_{\|}+I_{\perp}\right)$ the following relationship $r=2 P /(3-P)$ exists. The connection between the emission anisotropy $r$ (for polarized excitation) and the emission anisotropy $r_{\mathrm{n}}$ for the case of natural light excitation is: $r=2 r_{n}$ [2]. The corresponding expression for the degree of polarization $P$ and for $P_{n}$ is $P=2 P_{n} /\left(1-P_{n}\right)$ [3].
} 
counting method. Thus the measurements of the emission anisotropies of weakly fluorescing polar molecules with short lifetimes became possible in an external electric field, and the experimental results could be interpreted in terms of Liptay's theory [9].

\section{Basic equations of the analysis of dipole moments in the excited state}

General expression for the increment of the polarization degree, $\Delta P=P_{E}-P$ (where $P_{E}$ is the degree of polarization when the electric field is applied and $P-$ in the absence of the field), was obtained by Liptay [6] as a function of squared electric field intensity, which involves:

a) dipole moments and polarizabilities of solute molecules examined in the ground and excited states,

b) characteristics of the absorption and emission spectra,

c) the ratio of lifetimes $\tau_{\mathfrak{f}}$ and dielectric relaxation times $\tau_{\varepsilon}$.

In the case of molecules with sufficiently high dipole moments in the ground and excited states, the terms incorporating polarizability may be neglected. Then, on excitation by nonpolarized (natural) light perpendicular to the observation direction the following form of the Liptay formula was obtained [9]:

$$
\begin{array}{r}
\frac{\Delta P}{E_{\mathrm{ef}}^{2}}\left(2+\frac{9}{5 k_{\mathrm{A}} \tau_{\mathrm{f}}}\right)-\frac{9}{350 k_{\mathrm{A}} \tau_{\mathrm{f}}}\left(7-\frac{13}{9+10 k_{\mathrm{A}} \tau_{\mathrm{f}}}\right) \beta^{2} \mu_{\mathrm{g}}^{2}+\frac{\beta^{2} \mu_{\mathrm{e}}^{2}}{5\left(9+10 k_{\mathrm{A}} \tau_{\mathrm{f}}\right)} \\
-\frac{3}{5 k_{\mathrm{A}} \tau_{\mathrm{f}}}\left(1-\frac{33}{7\left(9+10 k_{\mathrm{A}} \tau_{\mathrm{f}}\right)}\right)\left\{\left[\left(\frac{\mathrm{d} \ln y}{\mathrm{~d} \tilde{\nu}}\right)_{\tilde{\nu}_{\mathrm{a}}}+\left(\frac{\mathrm{d} \ln \Phi}{\mathrm{d} \tilde{\nu}}\right)_{\tilde{\nu}_{\mathrm{e}}}\right] \frac{\beta}{h c}\left(\mu_{\mathrm{g}} \cdot \Delta \mu\right)\right. \\
\left.+\left[\left(\frac{1}{y} \frac{\mathrm{d}^{2} y}{\mathrm{~d} \tilde{\nu}^{2}}\right)_{\tilde{\nu}_{\mathrm{e}}}+\left(\frac{1}{\Phi} \frac{\mathrm{d}^{2} \Phi}{\mathrm{d} \tilde{\nu}^{2}}\right)_{\tilde{\nu}_{\mathrm{e}}}+2\left(\frac{\mathrm{d} \ln y}{\mathrm{~d} \tilde{\nu}}\right)_{\tilde{\nu}_{\mathrm{a}}}\left(\frac{\mathrm{d} \ln \Phi}{\mathrm{d} \tilde{\nu}}\right)_{\tilde{\nu}_{\mathrm{e}}}\right] \frac{(\Delta \mu)^{2}}{2 h^{2} c^{2}}\right\} \\
+\frac{12}{5\left(9+10 k_{\mathrm{A}} \tau_{\mathrm{f}}\right)}\left\{\left[\left(\frac{\mathrm{d} \ln y}{\mathrm{~d} \tilde{\nu}}\right)_{\tilde{\nu}_{\mathrm{a}}} \mu_{\mathrm{g}}+\left(\frac{\mathrm{d} \ln \Phi}{\mathrm{d} \tilde{\nu}}\right)_{\tilde{\nu}_{\mathrm{e}}} \mu_{\mathrm{e}}\right] \frac{\beta}{h c} \Delta \mu_{\mathrm{g}}\right. \\
+\left[\left(\frac{1}{y} \frac{\mathrm{d}^{2} y}{\mathrm{~d} \tilde{\nu}^{2}}\right)_{\tilde{\nu}_{\mathrm{e}}}+\left(\frac{1}{\Phi} \frac{\mathrm{d}^{2} \Phi}{\mathrm{d} \tilde{\nu}^{2}}\right)_{\tilde{\nu}_{\mathrm{e}}}\right] \frac{(\Delta \mu)^{2}}{\left.2 h^{2} c^{2}\right\}} \\
-\frac{1}{10 h^{2} c^{2}}\left(\frac{1}{\Phi} \frac{\mathrm{d}^{2} \Phi}{\mathrm{d} \tilde{\nu}^{2}}\right)_{\tilde{\nu}_{\mathrm{e}}}\left[3\left(m_{\mathrm{e}} \cdot \Delta \mu\right)^{2}-(\Delta \mu)^{2}\right]= \\
=\frac{\beta^{2}}{10}\left[3\left(m_{\mathrm{e}} \cdot \mu_{\mathrm{e}}\right)^{2}-\mu_{\mathrm{e}}^{2}\right]+\frac{\beta}{5 h c}\left(\frac{\mathrm{d} \ln \Phi}{\mathrm{d} \tilde{\nu}}\right)_{\tilde{\nu}_{\mathrm{e}}}\left[3\left(m_{\mathrm{e}} \cdot \mu_{\mathrm{e}}\right)\left(m_{\mathrm{e}} \cdot \Delta \mu\right)-\left(\mu_{\mathrm{e}} \cdot \Delta \mu\right)\right]
\end{array}
$$

where: $\Delta P=P_{E}-P$, 
$P_{E}, P$ - the degrees of polarization measured in the presence and absence of the electric field, respectively,

$E_{\text {ef }}$ - the effective Lorentz electric field,

$k_{\mathrm{A}}$ - the probability of reorientation of the luminescent molecules,

$\tau_{\mathfrak{f}}$ - the mean lifetime of molecules in the excited state,

$\beta=(k T)^{-1}, y=\epsilon / \tilde{\nu}$,

$\epsilon$ - the molar decadic extinction coefficient,

$\tilde{\nu}$ - the wavenumber ( $\mathrm{a}$ - absorption, $\mathrm{e}-$ fluorescence),

$\Phi$ - the relative fluorescence light intensity expressed in quanta per unit wavenumber interval,

$\Delta \mu=\mu_{\mathrm{e}}-\mu_{\mathrm{g}}$

$\mu_{\mathrm{e}}$ - the electric dipole moment in the excited state,

$\mu_{\mathrm{g}}$ - the electric dipole moment in the ground state,

$m_{\mathrm{e}}, m_{\mathrm{a}}$ - unit transition moment vectors in the emission and absorption, respectively.

The magnitude of $k_{\mathrm{A}} \tau_{\mathfrak{f}}$ can be obtained from the equation [9]:

$$
P=\left[3\left(m_{\mathrm{a}} \cdot m_{\mathrm{e}}\right)^{2}-1\right] /\left[7-\left(m_{\mathrm{a}} \cdot m_{\mathrm{e}}\right)^{2}+\frac{20}{3} k_{\mathrm{A}} \tau_{\mathrm{f}}\right],
$$

by measuring the degree of polarization of molecules placed in a rigid solution. For $k_{\mathrm{A}} \tau_{\mathrm{f}} \approx 0$, the magnitude of $\left(m_{\mathrm{a}} \cdot m_{\mathrm{e}}\right)^{2}$ is determined from the formula (2), and then from the same expression for fluid solution, $k_{\mathrm{A}} \tau_{\mathrm{f}}$ is obtained.

When the vectors $m_{\mathrm{e}}, \boldsymbol{\mu}_{\mathrm{g}}, \boldsymbol{\mu}_{\mathrm{e}}$ are assumed to be parallel for prolate and rigid molecules, the formula (1) can be employed to determine the dipole moments in the first excited singlet state. Liptay and co-workers [10] applied the following procedure to obtain the value of $\mu_{\mathrm{e}}$ : they measured the magnitude of $\Delta P / E_{\mathrm{ef}}^{2}$ for several wavelengths assuming $E_{\mathrm{ef}}=\left(\varepsilon_{\mathrm{DK}}+2\right) / 3 E$ to express the Lorentz effective field ( $\varepsilon_{\mathrm{DK}}$ is the permittivity), where $E$ denotes the external effective field. Provided with $k_{\mathrm{A}} \tau_{\mathrm{f}}$ for a given substance (from the formula (2)) and its spectral characteristics $y$ and $\Phi$, and following the initial estimation of $\mu_{\mathrm{e}}$ they calculated the values of the individual terms (six!) on the left-hand side of the equation (1). If the condition $k_{\mathrm{A}} \tau_{\mathrm{f}} \gg 1$ is satisfied, or for $k_{\mathrm{A}} \tau_{\mathrm{f}} \approx 1$, which is fulfilled for p-dimethylamino-p'-cyano-stilbene in dioxane, the sum of the components from 2 to 5 on the left-hand side of the equation (1) can be neglected compared to the first term. Ilence, the equation (1) can be written in the following simplified form:

$$
\frac{\Delta P}{E_{\text {ef }}^{2}}\left(2+\frac{9}{5 k_{\mathrm{A}} \tau_{\mathrm{f}}}\right)=A\left(\frac{\mathrm{d} \ln \Phi}{\mathrm{d} \tilde{\nu}}\right)_{\bar{\nu}_{\mathrm{e}}}+B
$$


where

$$
\begin{gathered}
A=0.4 \frac{\beta}{h c} \mu_{\mathrm{e}} \Delta \mu, \\
B=0.2 \beta^{2} \mu_{\mathrm{e}}^{2} .
\end{gathered}
$$

On substitution of the measured and calculated values into the eq. (3), and based on the formulae of the linear least square method, the direction coefficient $A$ and point $B$ of the intersection of the straight line (3) with the ordinate axis can be determined. Two values of $\mu_{\mathrm{e}}$ can be obtained from the formulae (4) and (5).

The following procedure has been applied in the present paper. Assuming $m_{\mathrm{e}}\left\|\mu_{\mathrm{g}}\right\| \mu_{\mathrm{e}}$, the eq. (1) can be reduced to a simple Newton binominal:

$$
a_{0}+a_{1} \mu_{\mathrm{e}}+a_{2}\left(\mu_{\mathrm{e}}\right)^{2}=0
$$

where

$$
\begin{aligned}
& a_{0}=\frac{\Delta P}{E_{\mathrm{ef}}^{2}}\left(2+\frac{9}{5 k_{\mathrm{A}} \tau_{\mathrm{f}}}\right)-\frac{9}{350 k_{\mathrm{A}} \tau_{\mathrm{f}}}\left(7-\frac{13}{9+10 k_{\mathrm{A}} \tau_{\mathrm{f}}}\right) \beta^{2} \mu_{\mathrm{g}}^{2} \\
& +\frac{3}{5 k_{\mathrm{A}} \tau_{\mathrm{f}}}\left(1-\frac{33}{7\left(9+10 k_{\mathrm{A}} \tau_{\mathrm{f}}\right)}\right)\left\{\left[\left(\frac{\mathrm{d} \ln y}{\mathrm{~d} \tilde{\nu}}\right)_{\tilde{\nu}_{\mathrm{a}}}+\left(\frac{\mathrm{d} \ln \Phi}{\mathrm{d} \tilde{\nu}}\right)_{\tilde{\nu}_{\mathrm{e}}}\right] \frac{\beta \mu_{\mathrm{g}}^{2}}{h c}\right. \\
& \left.-\left[\left(\frac{1}{y} \frac{\mathrm{d}^{2} y}{\mathrm{~d} \tilde{\nu}^{2}}\right)_{\tilde{\nu}_{\mathrm{a}}}+\left(\frac{1}{\Phi} \frac{\mathrm{d}^{2} \Phi}{\mathrm{d} \tilde{\nu}^{2}}\right)_{\tilde{\nu}_{\mathrm{e}}}+2\left(\frac{\mathrm{d} \ln y}{\mathrm{~d} \tilde{\nu}}\right)_{\bar{\nu}_{\mathrm{s}}}\left(\frac{\mathrm{d} \ln \Phi}{\mathrm{d} \tilde{\nu}}\right)_{\tilde{\nu}_{\mathrm{e}}}\right] \frac{\mu_{\mathrm{g}}^{2}}{2 h^{2} c^{2}}\right\}+ \\
& +\frac{12}{5\left(9+10 k_{\mathrm{A}} \tau_{\mathrm{f}}\right)}\left\{\left[\left(\frac{1}{y} \frac{\mathrm{d}^{2} y}{\mathrm{~d} \tilde{\nu}^{2}}\right)_{\tilde{\nu}_{\mathrm{a}}}+\left(\frac{1}{\Phi} \frac{\mathrm{d}^{2} \Phi}{\mathrm{d} \tilde{\nu}^{2}}\right)_{\tilde{\nu}_{\mathrm{e}}}\right] \frac{1}{2 h c}-\left(\frac{\mathrm{d} \ln y}{\mathrm{~d} \nu}\right)_{\tilde{\nu}_{\mathrm{a}}} \beta\right\} \frac{\mu_{\mathrm{g}}^{2}}{2 h c} \\
& -\frac{2 \mu_{\mathrm{g}}^{2}}{10 h^{2} c^{2}}\left(\frac{1}{\Phi} \frac{\mathrm{d}^{2} \Phi}{\mathrm{d} \tilde{\nu}^{2}}\right)_{\bar{\nu}_{\mathrm{e}}} \\
& a_{1}=-\frac{3}{\left.5 k_{\mathrm{A}} \tau_{\mathrm{f}}\right)}\left(1-\frac{33}{7\left(9+10 k_{\mathrm{A}} \tau_{\mathrm{f}}\right)}\right)\left\{\left[\left(\frac{\mathrm{d} \ln y}{\mathrm{~d} \tilde{\nu}}\right)_{\tilde{\nu}_{\mathrm{a}}}+\left(\frac{\mathrm{d} \ln \Phi}{\mathrm{d} \tilde{\nu}}\right)_{\tilde{\nu}_{\mathrm{e}}}\right] \frac{\beta \mu_{\mathrm{g}}}{h c}+\right. \\
& \left.-2\left[\left(\frac{1}{y} \frac{\mathrm{d}^{2} y}{\mathrm{~d} \tilde{\nu}^{2}}\right)_{\tilde{\nu}_{\mathrm{a}}}+\left(\frac{1}{\Phi} \frac{\mathrm{d}^{2} \Phi}{\mathrm{d} \tilde{\nu}^{2}}\right)_{\tilde{\nu}_{\mathrm{e}}}+2\left(\frac{\mathrm{d} \ln y}{\mathrm{~d} \tilde{\nu}}\right)_{\tilde{\nu}_{\mathrm{a}}}\left(\frac{\mathrm{d} \ln \Phi}{\mathrm{d} \tilde{\nu}}\right)_{\tilde{\nu}_{\mathrm{e}}}\right] \frac{\mu_{\mathrm{g}}}{2 h^{2} c^{2}}\right\} \\
& +\frac{12}{5\left(9+10 k_{\mathrm{A}} \tau_{\mathrm{f}}\right)}\left\{\left[\left(\frac{\mathrm{d} \ln y}{\mathrm{~d} \tilde{\nu}}\right)_{\tilde{\nu}_{\mathrm{a}}}-\left(\frac{\mathrm{d} \ln \Phi}{\mathrm{d} \tilde{\nu}}\right)_{\tilde{\nu}_{\mathrm{e}}}\right] \frac{\beta \mu_{\mathrm{g}}}{h c}\right. \\
& \left.+\left[\left(\frac{1}{y} \frac{\mathrm{d}^{2} y}{\mathrm{~d} \tilde{\nu}^{2}}\right)_{\tilde{\nu}_{\mathrm{a}}}+\left(\frac{1}{\Phi} \frac{\mathrm{d}^{2} \Phi}{\mathrm{d} \tilde{\nu}^{2}}\right)_{\tilde{\nu}_{\mathrm{e}}}\right] \frac{\mu_{\mathrm{g}}}{h^{2} c^{2}}\right\} \\
& +\frac{2 \mu_{\mathrm{g}}}{5 h c}\left[\frac{1}{h c}\left(\frac{1}{\Phi} \frac{\mathrm{d}^{2} \Phi}{\mathrm{d} \tilde{\nu}^{2}}\right)_{\tilde{\nu}_{\mathrm{e}}}+\beta\left(\frac{\mathrm{d} \ln \Phi}{\mathrm{d} \tilde{\nu}}\right)_{\tilde{\nu}_{\mathrm{e}}}\right] \text {, }
\end{aligned}
$$




$$
\begin{gathered}
a_{2}=\frac{12}{5\left(9+10 k_{\mathrm{A}} \tau_{\mathrm{f}}\right)}\left\{\frac{\beta^{2}}{12}+\left(\frac{\mathrm{d} \ln \Phi}{\mathrm{d} \tilde{\nu}}\right)_{\tilde{\nu}_{\mathrm{e}}} \frac{\beta}{h c}\right. \\
\left.+\left[\left(\frac{1}{y} \frac{\mathrm{d}^{2} y}{\mathrm{~d} \tilde{\nu}^{2}}\right)_{\tilde{\nu}_{\mathrm{e}}}+\left(\frac{1}{\Phi} \frac{\mathrm{d}^{2} \Phi}{\mathrm{d} \tilde{\nu}^{2}}\right)_{\tilde{\nu}_{\mathrm{e}}}\right] \frac{1}{2 h^{2} c^{2}}\right\}-\frac{1}{5 h^{2} c^{2}}\left(\frac{1}{\Phi} \frac{\mathrm{d}^{2} \Phi}{\mathrm{d} \tilde{\nu}^{2}}\right)_{\tilde{\nu}_{\mathrm{e}}} \\
-\frac{3}{\left.5 k_{\mathrm{A}} \tau_{\mathrm{f}}\right)}\left(1-\frac{33}{7\left(9+10 k_{\mathrm{A}} \tau_{\mathrm{f}}\right)}\right)\left[\left(\frac{1}{y} \frac{\mathrm{d}^{2} y}{\mathrm{~d} \tilde{\nu}^{2}}\right)_{\tilde{\nu}_{\mathrm{a}}}+\left(\frac{1}{\Phi} \frac{\mathrm{d}^{2} \Phi}{\mathrm{d} \tilde{\nu}^{2}}\right)_{\tilde{\nu}_{\mathrm{e}}}\right. \\
\left.+2\left(\frac{\mathrm{d} \ln y}{\mathrm{~d} \tilde{\nu}}\right)_{\tilde{\nu}_{\mathrm{a}}}\left(\frac{\mathrm{d} \ln \Phi}{\mathrm{d} \tilde{\nu}}\right)_{\tilde{\nu}_{\mathrm{e}}}\right] \frac{1}{2 h^{2} c^{2}}-\frac{\beta^{2}}{5}-\frac{2 \beta}{5 h c}\left(\frac{\mathrm{d} \ln \Phi}{\mathrm{d} \tilde{\nu}}\right)_{\tilde{\nu}_{\mathrm{e}}} \cdot
\end{gathered}
$$

Thus, $\mu_{\mathrm{e}}$ can be determined from equations (6)-(9) by a single measurement of $\Delta P / E_{\text {ef }}^{2}$. Irrespective of a complicated form of equations (6)-(9) occurring in Liptay's theory, such an approach to the determination of $\mu_{\mathrm{e}}$ became possible thanks to modern computation techniques employed.

\section{Experimental}

\subsection{Materials}

The effect of the external electric field on the emission anisotropy of the following prolate donor-acceptor substituted trans-stilbenes was investigated:

4-hydrogen-4'-diphenylphosphinyl-stilbene (1a),

4-dimethylamino-4'-diphenylphosphinyl-stilbene ( $\underline{1 \mathrm{~b}})$,

4-dimethylamino-4'-cyano-stilbene (1g),

4-dimethylamino-4'-bromo-stilbene (1h),

4-dimethylamino-4'-chloro-stilbene (11),

4-dimethylamino-4'-fluoro-stilbene ( $\underline{1 \mathrm{j}})$,

4-dimethylamino-4'-methoxy-stilbene (1k),

4-dimethylamino-4'-nitro-stilbene (11),

4-amino-4'-nitro-stilbene $(\underline{1 \mathrm{~m}})$,

4-dimethylamino-4'-isocyanate-stilbene $(\underline{1 \mathrm{n}})$.

Cyclohexane used as a nonpolar solvent was specially purified to remove benzene residues and other tracer impurities, the presence of which could result in an increase of conductivity in the solution and, hence, in possible breakdown in the solution layer. Similar purification procedure was applied to n-heptane.

In order to determine the value of $k_{\mathrm{A}} \tau_{\mathrm{f}}$ (occurring in eq. (2)), the substances investigated were placed in polyvinyl alcohol (PVA) films by the method of Tanizaki [13]. 


\subsection{Methods}

The absorption spectra were measured using an automatic double-beam Specord M40 (C. Zeiss Jena) spectrophotometer. The emission spectra were recorded by means of a standard apparatus designed for measuring the fluorescence spectra, with a $250 \mathrm{~W}$ xenon lamp as an excitation source, SPM-2 exciting and analyzing monochromators, and a Thorn EMI 6255 photomultiplier. The measurement was fully automatic and controlled by a microcomputer. Light intensity was measured for a single wavelength of the observed light by means of a V-533 voltmeter, and repeated 20 times. The results were averaged and recorded on a floppy disk after the measurement had been completed.

The employment of two-channel photon counting method $[14,15]$ with the use of Wollaston prism for the measurement of emission anisotropy on continuous excitation enabled the elimination of disadvantages typical for modulation methods and facilitated significantly the manual service during the experiment. Owing to the simplicity of the electronic part of the apparatus, the method is not affected by different kinds of instabilities in external parameters such as temperature, supply voltage, etc. The employment of stepper motors (moving all mobile optical elements), an IEC-625 interface monitoring the operation of a system programmable EMG 777D (Hungary) graphic calculator enabled fully automatic measurements.

A schematic diagram of the apparatus measuring the emission anisotropy by the photon-counting method has been given and discussed in detail by Kubicki [16].

The detection of the effect of external electric field on the emisssion anisotropy required the use of a special highvoltage cell which was constructed according to Czekalla's design [3], thus satisfying the following conditions:

a) high resistance of insulation (teflon casing),

b) chemical neutrality (stainless steel, teflon),

c) possibility of temperature stabilization (thermostated electrodes),

d) resistance to possible breakdowns in the solution and, hence, to rapid increase of pressure inside the cell (Wood horn intended to capture superfluous light opposite the window through which fluorescence is observed, operating simulteneously as a safety valve),

e) the possibility to observe light in homogeneous region of the electric field (the limitation of the observation field by a suitable diaphragm and special shape of electrodes).

Nonpolarized light was also used for excitation in order to increase the intensity of the observed light. 
$\sqrt{9}$

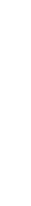

1

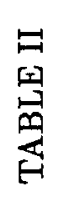

胥

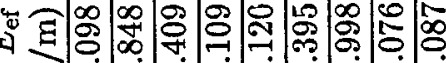

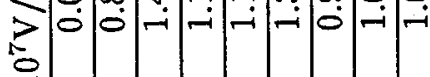

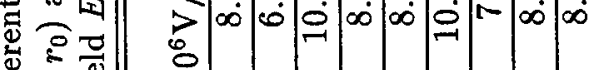

过

\%7

क

至.

I $\quad$ D

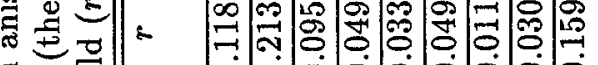

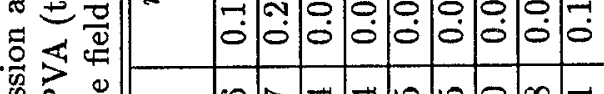

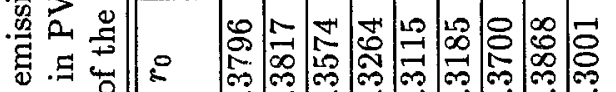

ปี

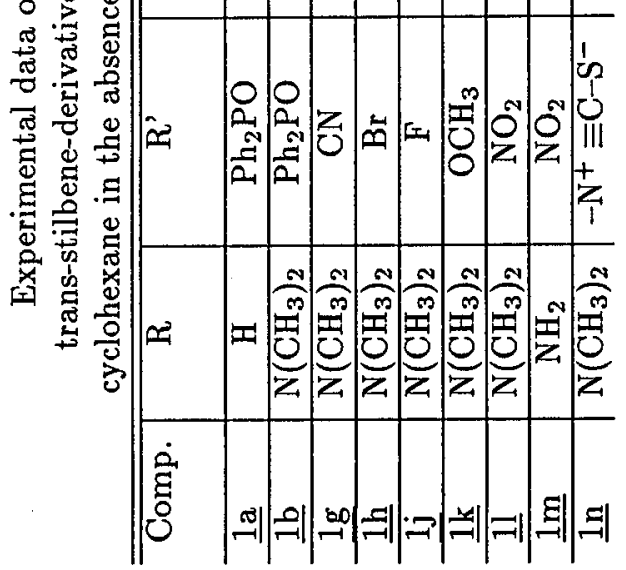

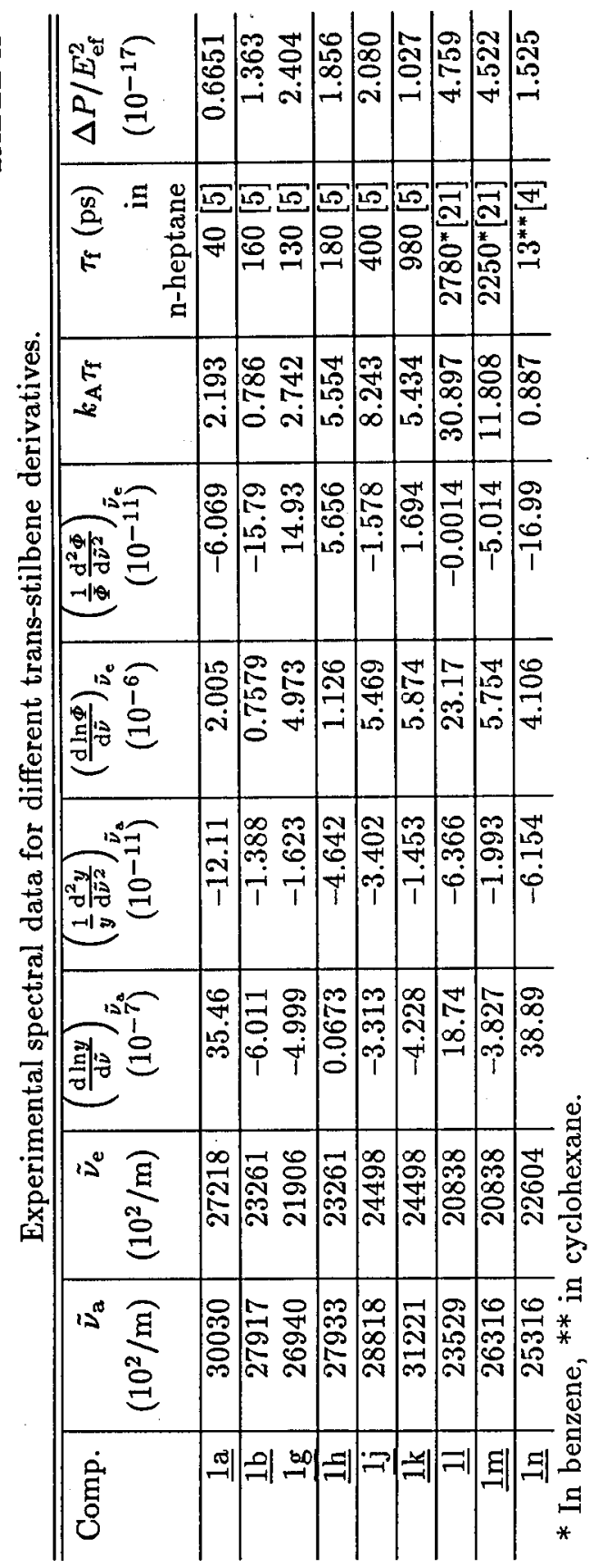




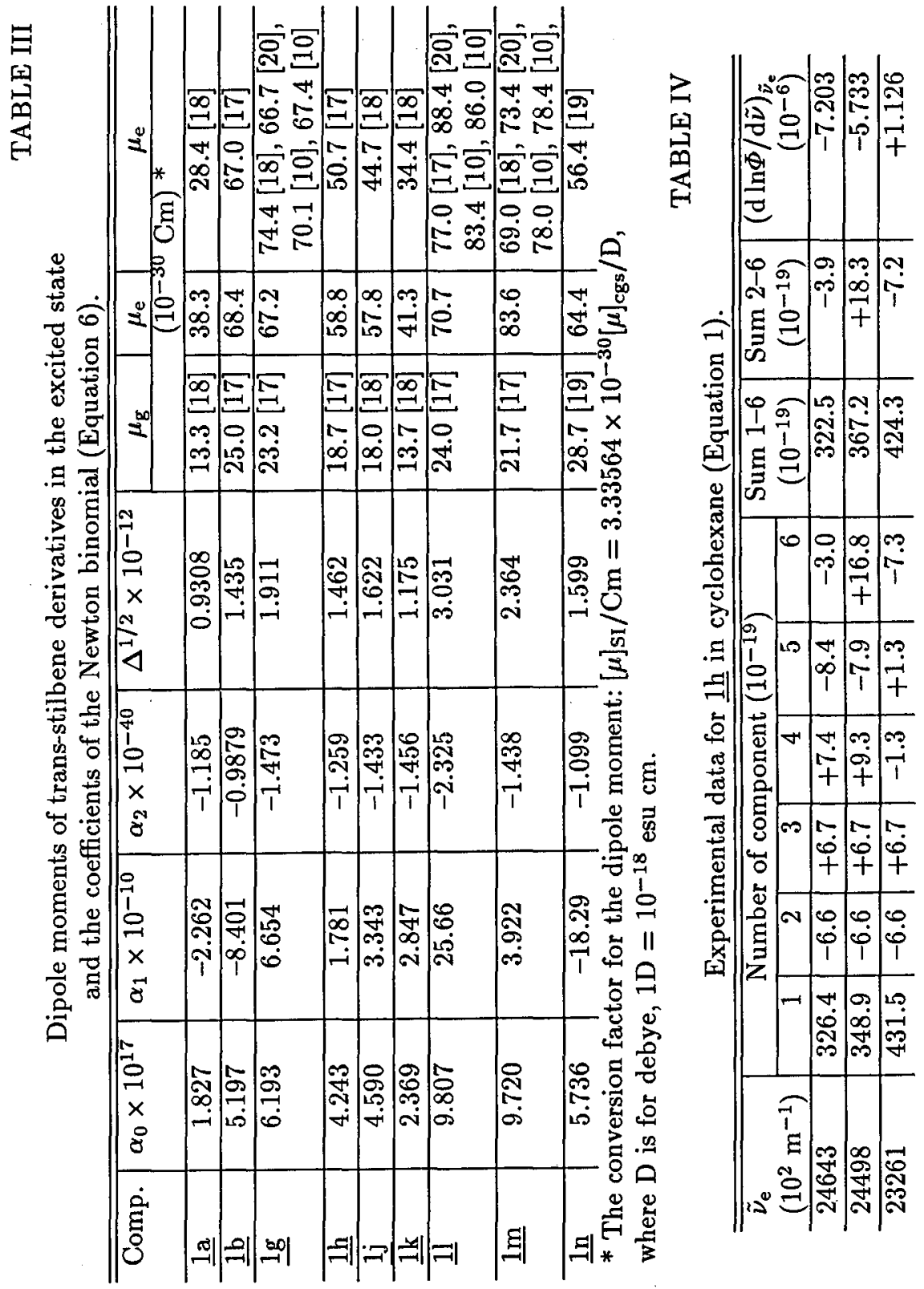




\section{Results and discussion}

An example of long-wave absorption and fluoroscence spectra is shown in Fig. 1 for compound 1a in cyclohexane. The wave numbers corresponding to emis-
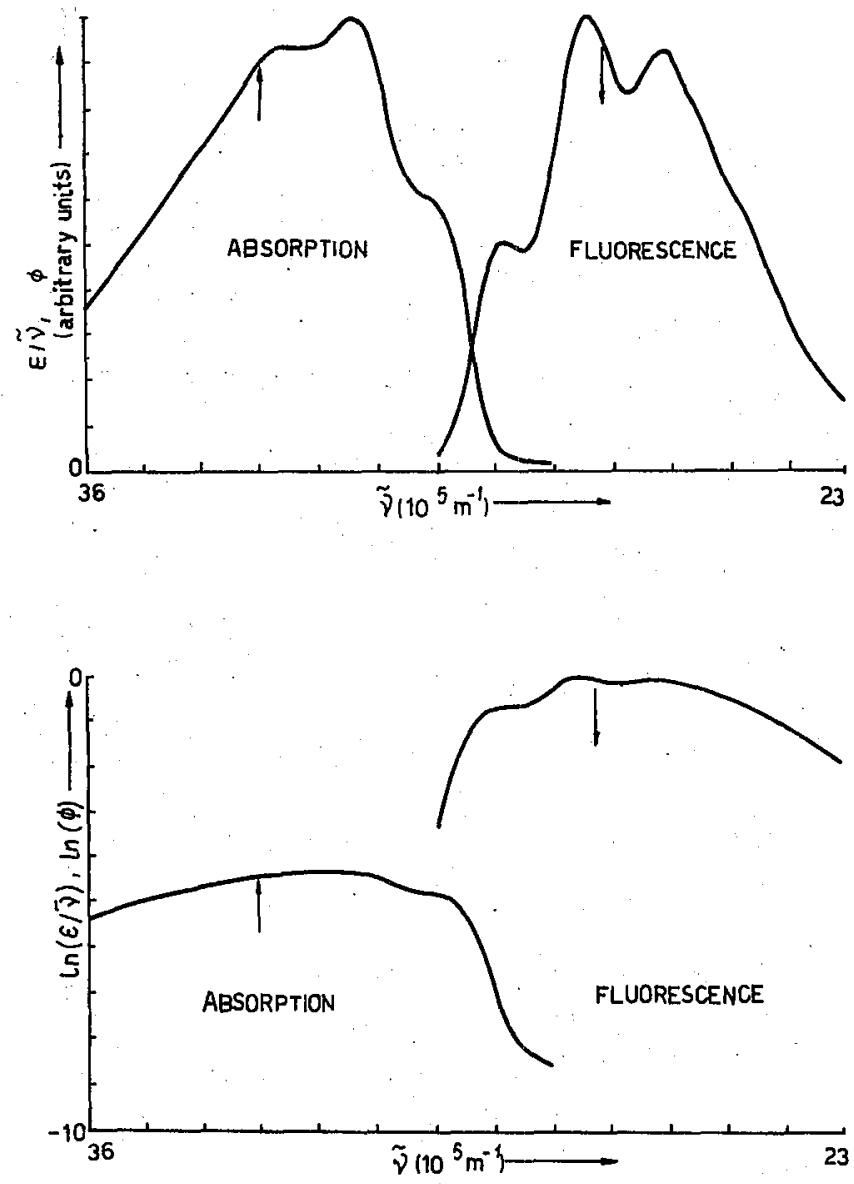

Fig. 1. Absorption $\varepsilon / \tilde{\nu}$ and emission $\Phi$ spectra and their logarithms for compound $\underline{1 \mathrm{a}}$ in cyclohexane.

sion anisotropy measurements have been indicated on the curves by arrows. Table I summarizes the resulting emission anisotropy values $r_{0}$ measured for compounds investigated in rigid PVA solutions, and also $r$ and $r_{E}$ obtained in cyclohexane in the absence and presence of the electric field, respectively. Spectral characteristics determined from the spectra are given in Table II. The values of these derivatives, depending only on the shape of the absorption and emission curves, were determined numerically. The value of product $k_{\mathrm{A}} \tau_{\mathrm{f}}$ was found from the formula (2) by 
the method of Liptay. In the calculations of the Lorentz effective electric field, the permittivity of cyclohexane $\varepsilon_{\mathrm{DK}}=2.023$ was assumed (Table I). Based on the calculation results given in Table II and on the formulae (7)-(9), the coefficients of quadratic binomial (6) were calculated. The values of these coefficients and the discriminants are given in Table III. The values of electric dipole moments in the excited state $\mu_{\mathrm{e}}$, thus obtained for known [17-19] dipole moments in the ground state, $\mu_{\mathrm{g}}$, have also been given in Table III.

In order to verify such a simplified method of determining the values of the dipole moments $\mu_{\mathrm{e}}$ in the excited state, $\mu_{\mathrm{e}}$ was also determined by the method of Liptay $[9,10]$ for one of the substances investigated and denoted by $1 \mathrm{~h}$. The components occuring in the equation (1), calculated for $\tilde{\nu}_{\mathrm{a}}=27933 \times 10^{2} \mathrm{~m}^{-1}$, $k_{\mathrm{A}} \tau_{\mathrm{f}}=5.554$, and for three different wave numbers $\tilde{\nu}_{\mathrm{e}}$, have been given in Table IV. The respective errors resulting when neglecting terms $2-6$ amount to $-1.2 \%,+5 \%$, $-1.7 \%$ of the value of complete left-hand side of the equation (1). Next, referring the values of the left-hand side of the equation (1), excluding terms 2-6, to the ordinate axis, and $(\mathrm{d} \ln \Phi / \mathrm{d} \tilde{\nu})_{\tilde{\nu}_{e}}$ to the abscissae axis (Table IV), the parameters of the equation (3) were determined by the least square method: $A=1.244 \times 10^{-12}$, $B=4.179 \times 10^{-17}$, the correlation coefficient amounting to 0.999 . Hence, based on the equations (4) and (5), $\mu_{\mathrm{e} A}=60.5 \times 10^{-30} \mathrm{Cm}$ and $\mu_{\mathrm{e} B}=58.9 \times 10^{-30} \mathrm{Cm}$ were obtained which are in very good accordance with the value of $\mu_{\mathrm{e}}$ determined from the equation (6) (see Table III).

Table III summarizes also the values of dipole moments $\mu_{\mathrm{e}}$ determined by the solvent method [17-19], by dichroism [20], and by Czekalla's method of polarization in electric field $[6,10]$. Considerable differences have been observed for molecules $\underline{1 \mathrm{a}}, \underline{\mathrm{h}}, 1 \mathrm{j}, \underline{\mathrm{k}}, \underline{\mathrm{m}}, \underline{1 \mathrm{n}}$, which can be accounted for by the fact that in Liptay's equations (1), (3), and (6), the effective electric field was represented by the Lorentz field whereas in the solvatochromic method by the Onsager field. In order to compare the values of $\mu_{\mathrm{e}}$ obtained, they should be determined from the formulae (6)-(9) assuming $E_{\text {ef }}=E$, and then corrected using the method proposed by Baumann [22] for an appropriate type of the electric field. Thus, the dipole moment $\mu_{\mathrm{e}}^{\circ}$ of a free molecule in an excited state is obtained similarly as when employing the solvatochromic method.

Based on the theoretical considerations of Baumann and Deckers [22] and applying the Onsager model for a spherical cavity [23] and the approximation $\left(\alpha / a^{3}\right)\left(1 /\left(4 \pi \varepsilon_{0}\right)\right)=\frac{1}{2}$ (where $\alpha$ is the polarizability, $a-$ the Onsager interaction radius of the solute, and $\varepsilon_{0}$ - the permittivity of vacuum [17], the following expression is obtained for luminescent molecules in nonpolar solvent (the experiments were carried out in cyclohexane with permittivity $\varepsilon_{\mathrm{DK}}=2.023$ at $T=295$ K):

$$
\mu_{\mathrm{e}}^{\prime}=\frac{3 \varepsilon_{\mathrm{DK}}}{\varepsilon_{\mathrm{DK}}+2} \mu_{\mathrm{e}}^{\circ} \approx 1.509 \mu_{\mathrm{e}}^{\circ}
$$

Applying the Lorentz approximation one obtains:

$$
\mu_{\mathrm{e}}^{\prime}=\frac{\varepsilon_{\mathrm{DK}}+2}{3} \mu_{\mathrm{e}}^{0} \approx 1.341 \mu_{\mathrm{e}}^{\circ} .
$$

Electric dipole moments $\mu_{\mathrm{e}}^{\prime}$ determined form the formulae (6)-(9) for $E_{\mathrm{ef}}=E$, 
and the corrected values of $\mu_{\mathrm{e}}^{\circ}$, based on the expressions (10) and (11), have been given in Table $\mathrm{V}$.

Corrected dipole moments $\mu_{e}^{\circ}$

TABLE V

\begin{tabular}{c|c|c|c}
\multicolumn{4}{c}{ of trans-stilbene derivatives in the excited state. } \\
\hline \hline \multirow{2}{*}{ Compound } & $\mu_{\mathrm{e}}^{\prime}$ Eq. (6) & $\mu_{\mathrm{e}}^{\circ}$ Eq.(10) & $\mu_{\mathrm{e}}^{\circ}$ Eq. (11) \\
\cline { 2 - 4 } & \multicolumn{3}{|c}{$\left(10^{-30} \mathrm{C} \mathrm{m}\right)$} \\
\hline$\underline{1 \mathrm{a}}$ & 52.0 & 34.5 & 38.8 \\
\hline$\underline{\mathrm{b}}$ & 95.8 & 63.5 & 71.4 \\
\hline$\underline{\mathrm{g}}$ & 89.8 & 59.5 & 67.0 \\
\hline$\underline{\mathrm{h}}$ & 78.8 & 52.2 & 58.8 \\
\hline$\underline{1 \mathrm{j}}$ & 77.2 & 51.2 & 57.6 \\
\hline$\underline{\mathrm{k}}$ & 55.2 & 36.6 & 41.2 \\
\hline$\underline{1 \mathrm{l}}$ & 92.8 & 61.5 & 69.2 \\
\hline$\underline{\mathrm{m}}$ & 111.7 & 74.0 & 83.3 \\
\hline$\underline{1 \mathrm{n}}$ & 90.4 & 59.9 & 67.4
\end{tabular}

The comparison of the excited state dipole moments $\mu_{\mathrm{e}}^{\circ}$ determined from the Liptay's theory and corrected by the use of the formula (10) with respective values obtained from solvatochromic effect reveals much better agreement.

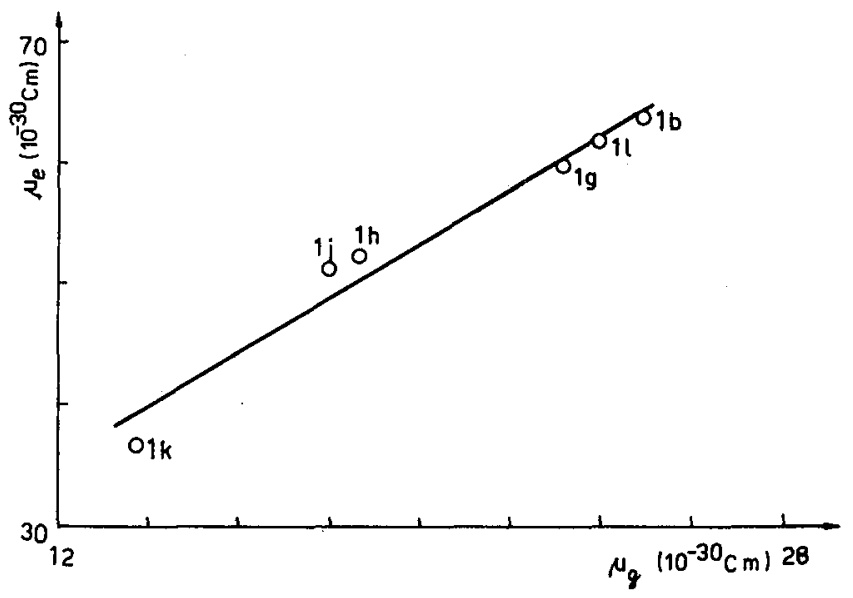

Fig. 2. Dependence of the value of $\mu_{\mathrm{e}}$ on $\mu_{\mathrm{g}}$ for para-disubstituted trans-stilbenes with the same donor $R=\mathrm{N}\left(\mathrm{CH}_{3}\right)_{2}$ and different acceptors $R$ '.

Para-disubstituted trans-stilbenes investigated, while displaying relatively high fluorescence quantum yields, have often very short mean lifetimes of about $100 \mathrm{ps}$ (Table II). Rotational relaxation times of these molecules in many solvents of low viscosities are comparable to their mean lifetimes in the first excited 
singlet state. This results in high fluorescence anisotropy observed in these solvents. The magnitude of the electric charge displacement and the concomitant change in electric dipole moment relative to that of unsubstituted stilbene depends, for 4, 4'-disubstituted stilbenes, on the electron-donor-acceptor properties of the substituents. It is of particular interest to compare the values of the excited state dipole moments of para-substituted trans-stilbenes with the same substituent $\mathrm{R}=\mathrm{N}\left(\mathrm{CH}_{3}\right)_{2}$ in position 4 (Table I) and different acceptors $\mathrm{R}$ '. The total electronic interaction of substituent $R^{\prime}$ in position $4^{\prime}$ with the remaining $\pi$-electronic system in the ground and excited state can be expressed by the Hammett constants $\sigma_{p}$ [18]. Therefore, for enhancing electron properties of acceptor $R$ ' for a certain donor substituent $R$, the values of the dipole moment in the ground $\mu_{\mathrm{g}}$ and in the excited state $\mu_{\mathrm{e}}$ increase. There exists a linear relation between the values of $\mu_{\mathrm{e}}$ and $\mu_{\mathrm{g}}$ (Fig. 2).

\section{Acknowledgments}

We thank Ms Renata Basis for technical assistance in the preparation of this work.

\section{References}

[1] A. Jabłoński, Bull. Acad. Pol. Sci., Ser. sci. math. astr. et phys. 8, 259 (1960).

[2] A. Kawski, Bull. Acad. Pol. Sci., Ser. sci. math., astr. et phys. 6, 671 (1958).

[3] S.I. Vavilov, W.L. Levshin, Z. Phys. 16, 135 (1923).

[4] A. Kawski, M. Alicka, Z. Naturforsch. 34a, 1371 (1979); 35a, 775 (1980).

[5] A. Kawski, M. Alicka, D. Gloyna, Z. Naturforsch. 36a, 1259 (1981).

[6] J. Czekalla, Z. Elektrochem. 64, 1221 (1960).

[7] J. Czekalla, Chimia, 15, 26 (1961).

[8] G. Weber, J. Chem. Phys. 43, 521 (1965).

[9] W. Liptay, Z. Naturforsch. 18a, 705 (1963).

[10] J. Czekalla, W. Liptay, K.-O. Meyer, Ber. Bunsenges. Phys. Chem. 67, 465 (1963).

[11] G. Weil, C. Hornick, Biopolymers 10, 2029 (1971).

[12] S. Sokerov, G. Weil, Biophys. Chem. 10, 161 (1979).

[13] Y. Tanizaki, Bull. Chem. Soc. Japan 32, 75 (1959).

[14] E.J. Darland, G.E. Leroi, C.G. Enke, Anal. Chem. 51, 240 (1979).

[15] E.J. Darland, J.E. Hornshuh, C.G. Enke, G.E. Leroi, Anal. Chem. 51, 245 (1979).

[16] A. Kubicki, Exp. Tech. Phys. 37, 329 (1989).

[17] A. Kawski, I. Gryczyński, Ch. Jung, K.-H. Heckner, Z. Naturforsch. 32a, 420 (1977). 
[18] I. Gryczyński, D. Gloyna, A. Kawski, Z. Naturforsch. 35a, 777 (1980).

[19] I. Gryczyński, A. Kawski, Ch. Jung, I. Janić, Z. Naturforsch. 37a, 259 (1982).

[20] W. Liptay, J. Czekalla, Z. Elektrochem. 65, 721 (1961).

[21] A. Kawski, J. Kamiński, J. Kukielski, Z. Naturforsch. 34a, 702 (1979).

[22] W. Baumann, H. Deckers, Ber. Bunsenges. Phys. Chem. 81, 786 (1977).

[23] L. Onsager, J. Chem. Phys. 58, 1486 (1936). 